\title{
Surface Reconstruction from Sparse Fringe Contours
}

\author{
G. Cong and B. Parvin \\ Information and Computing Sciences Division \\ Lawrence Berkeley National Laboratory \\ Berkeley, CA 94720
}

\begin{abstract}
A new approach for reconstruction of 3D surfaces from 2D cross-sectional contours is presented. By using the so-called "Equal Importance Criterion," we reconstruct the surface based on the assumption that every point in the region contributes equally to the surface reconstruction process. In this context, the problem is formulated in terms of a partial differential equation (PDE), and we show that the solution for dense contours can be efficiently derived from distance transform. In the case of sparse contours, we add a regularization term to insure smoothness in surface recovery. The proposed technique allows for surface recovery at any desired resolution. The main advantage of the proposed method is that inherent problems due to correspondence, tiling, and branching are avoided. Furthermore, the computed high resolution surface is better represented for subsequent geometric analysis. We present results on both synthetic and real data.
\end{abstract}

\section{Introduction}

The problem of reconstructing a 3D surface from a set of $2 D$ cross sectional contours is an important one in diverse scientific fields. For example, CT and MRI techniques can provide dense serial section representation of electron density and water molecule concentration at different locations along a particular axis. Similarly, in confocal microscopy, cross sections are obtained by focusing the optical system at specific locations along the $z$ axis. This type of data defines the intersections of the object surface with a plane along a desired orientation. We plan to utilize these cross sections and recover the $3 \mathrm{D}$ surfaces of the object for visualization as well as geometric analysis.

The main idea behind existing techniques is to use small triangles to connect contours in adjacent sections. This approach leads to three sources of ambiguities: (1) correspondence, (2) tiling, and (3) branching problems. The correspondence problem involves finding the correct connection between adjacent contours $[4,6,7,18,24]$. The tiling problem involves utilizing slice chords for constructing triangles in the regions between contours $[13,11,23,8,12,5]$. The

*This work is supported by the Director, Office of Energy Research, Office of Computation and Technology Research, Mathematical. Information, and Computational Sciences Division of the U.S. Department of Energy under contract No. DE-AC03-76SF00098 with the University of California. The LBNL publication number is 42163. E-mail: gcong@george.lbl.gov or parvin@george.lbl.gov main issue being how to find optimal tiling and how to account for topological changes. The branching problem occurs when a contour in one slice can correspond to more than one contour in an adjacent slice, e.g., bifurcation in the $3 \mathrm{D}$ shape $[14,18,8,5]$. Most of the existing techniques focus (at most) on resolving one or two of these problems.

In this paper, we treat the problem in a entirely new way by computing every point on the surface directly. This is based on representing the problem as a partial differential equation followed by a simple linear solution for dense contours and a regularized solution for sparse contours. In this sense, we can compute the coordinates of every point on the surface to construct a high resolution map of the $3 \mathrm{D}$ data of which the 0 value isosurface corresponds to the object surface. Isosurfaces have the advantage that subsequent geometric analysis of the object can be easily carried out $[19,20]$. As a result, our solution naturally avoids the correspondence, tiling, and branching problems in favor of a more robust solution. The underlying constraint is based on the Equal Importance Criterion (EIC), which suggests that all points contribute equally to the shape reconstruction process. Formally, the constraint states that surface height decreases linearly along the trajectory of its gradient, and as a result, the problem reduces to solving a PDE. This PDE has a simple linear solution for dense contours. However, a regularized approach is needed for sparse contours. Experimental results on both synthetic and contours obtained from real images are included.

\section{Preliminary definition}

Let $\mathcal{S}$ represent a 3D surface with $C\left(h_{i}\right)$, and $i=1, \ldots, m$ as the cross-sectional contours which may be defined as the intersections of $\mathcal{S}$ with a series of planes $z=h_{i}$. Without losing generality, we assume that $h_{i}=i, i=1, \ldots, m$. Let the projection of $C\left(h_{i}\right)$ on the $\mathrm{x}-\mathrm{y}$ ( or $z=h_{i}$ ) be $\mathcal{C}_{i}, i=$ $1, \ldots, m$. The problem is: Given $\mathcal{C}_{i} i=1, \ldots, m$, find the surface $\mathcal{S}$ such that $\mathcal{C}_{i}$ are the intersections of $\mathcal{S}$ with the plane $z=i$.

\section{Equal Importance Criterion}

The proposed reconstruction problem is underconstrained and ill-posed. To constrain the problem, we impose a smoothness measure based on EIC. Consider a simple example of Figure 1a with a pair of contours corresponding to $\mathcal{C}_{1}$ and $\mathcal{C}_{2}$. In order to define a region $R\left(\mathcal{C}_{1}, \mathcal{C}_{2}\right)$, let $R_{\mathcal{C}_{i}}^{\prime}(x, y)$ be a function of $\mathcal{C}_{i}$ such that:

$$
R_{\mathcal{C}_{i}}^{\prime}(x, y)= \begin{cases}-1, & \text { if }(x, y, i)(x, y) \text { is inside } \mathcal{C}_{i} \\ 1, & \text { if }(x, y) \text { is outside of } \mathcal{C}_{i} \\ 0, & \text { if }(x, y) \in \mathcal{C}_{i}\end{cases}
$$


$R\left(\mathcal{C}_{1}, \mathcal{C}_{2}\right)$ is now defined as (see Figure1):

$$
R\left(\mathcal{C}_{1}, \mathcal{C}_{2}\right)=\left\{(x, y) \mid R_{\mathcal{C}_{1}}^{\prime}(x, y) R_{\mathcal{C}_{2}}^{\prime}(x, y) \leq 0\right\}
$$

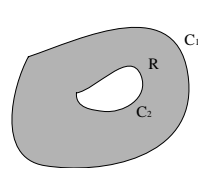

(a)

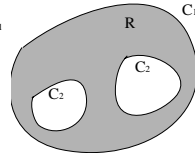

(b)

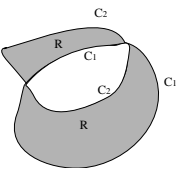

(c)

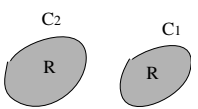

(d)
Figure 1: $\quad R\left(\mathcal{C}_{1}, \mathcal{C}_{m}\right)$. (a) Doubly connected region. (b) Multiply connected region. (c) Intersection. (d) Isolated objects.

In $R\left(\mathcal{C}_{1}, \mathcal{C}_{2}\right)$, we want to construct a surface $f(x, y)$ such that, say, $f\left(\mathcal{C}_{1}\right)=1, f\left(\mathcal{C}_{2}\right)=2$. Obviously in the absence of any constraints, infinitely many solutions exist. To constrain the problem, we assert that every point in $\mathcal{R}\left(\mathcal{C}_{1}, \mathcal{C}_{2}\right)$ is equally important and contributes similarly to the reconstruction process. Any other assumption means that we know something about the surface. We call this the Equal Importance Criterion. This constraint is formalized by requiring that the change in the gradient-magnitude along the gradient direction should be zero, that is:

$$
\mathcal{J}_{1}(f)=\nabla(|\nabla f|) \cdot \frac{\nabla f}{|\nabla f|}=0
$$

where $\nabla f$ indicates the gradient of $f, \|$ the norm and . the inner product. The above PDE implies that along each trajectory of the gradient of the surface, the magnitude of the gradient is a constant. In another words, the height decreases linearly from 2 to 1 . Thus, in view of height, which is our only clue about the surface, all points are equally important to us. $\mathcal{J}_{1}$ can be reduced to:

$$
\mathcal{J}_{2}(f)=f_{x}^{2} f_{x x}+2 f_{x} f_{y} f_{x y}+f_{y}^{2} f_{y y}=0
$$

where subscript indicates derivative, such as $f_{x}=$ $\frac{\partial f}{\partial x}, f_{x y}=\frac{\partial^{2} f}{\partial x \partial y}$. Thus, the problem becomes:

Find $f(x, y), \quad(x, y) \in R\left(\mathcal{C}_{1}, \mathcal{C}_{2}\right)$,

such that $\mathcal{J}_{2}(f)=0, \quad f\left(\mathcal{C}_{1}\right)=1, \quad f\left(\mathcal{C}_{2}\right)=2$.

Equation (4) is called the "Infinity Laplacian" (see Appendix A), which has been studied in the literature $[1,2,3,10]$. Equation (4) has several important properties [2]. These are:

1. There is at most one solution of the Dirichlet's problem for $f$, which may not be a smooth solution for any $\mathcal{C}_{1}$ and $\mathcal{C}_{2}$. But if we redefine the "solution" in a suitable weak sense, then a solution does exist.

2. The trajectory of the vector field for $\nabla f$ is either a convex curve or a straight line, and the solution is infinitely differentiable with convex curves.

\section{Surface reconstruction}

The proposed shape reconstruction, from cross sections, has several advantages:

1. The correspondence, tiling, and branching problems have been eliminated, and as we will see later, an efficient solution can be easily obtained.
2. The distance between $C_{1}$ and $C_{2}$ in the $z$ direction is no longer important because it only changes the solution by a scale.

3. The surface is smooth at most locations because a weak solution is guaranteed. Furthermore, at a singular point-if it exists-the surface is at least continuous to $C^{1}$.

In the rest of this section, we outline the details of solution to Equation (5) and show how it can be extended to other types of contours as well.

\subsection{Solving the PDE}

Many numerical methods can be used to solve Equation (5), and at least a weak solution is guaranteed. However, we show that a more straightforward and efficient approximation possible.

Let's define $\mathcal{D}_{\mathcal{C}}(x, y)$ as the Distance Transformation (chamfer image) of curve $\mathcal{C}$, where $\mathcal{D}_{\mathcal{C}}(x, y)$ has the same sign of $R_{\mathcal{C}}^{\prime}(x, y)$. For each point $p$ (shown in Figure 2), there should be a gradient trajectory $\gamma$ passing through it such that it intersects $\mathcal{C}_{1}$ and $\mathcal{C}_{2}$ at $p_{1}$ and $p_{2}$, respectively. Since $\mathcal{C}_{1}$ and $\mathcal{C}_{2}$ are equal height contours, it is easy to show that the normal of these two contours and the gradient of surface are in the same direction [22]. Thus $\gamma \perp \mathcal{C}_{1}$ at $p_{1}$ and $\gamma \perp \mathcal{C}_{2}$ at $p_{2}$. We can approximate the curve $\gamma$, passing through $p$, by drawing two line segments $p p_{1}^{\prime} \perp \mathcal{C}_{1}, p p_{2}^{\prime} \perp$ $\mathcal{C}_{2}$, to create $p_{1}^{\prime} p p_{2}^{\prime}$. Let $l$ denote the length of $\gamma$ from $p_{1}$ to $p_{2}$. Hence, $l \approx\left|p_{1}^{\prime} p\right|+\left|p_{2}^{\prime} p\right|$. The preceding formulation indicates that $\left|p_{1}^{\prime} p\right|=-\mathcal{D}_{\mathcal{C}_{1}}(p),\left|p_{2}^{\prime} p\right|=\mathcal{D}_{\mathcal{C}_{i+1}}(p)$. Since the height decreases linearly. $f$ can be set to:

$$
f(x, y)=\frac{\mathcal{D}_{\mathcal{C}_{2}}(x, y)-2 \mathcal{D}_{\mathcal{C}_{1}}(x, y)}{\mathcal{D}_{\mathcal{C}_{2}}(x, y)-\mathcal{D}_{\mathcal{C}_{1}}(x, y)}
$$

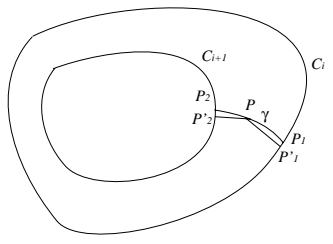

(a)

Figure 2: Initialization of $f(x, y)$ from chamfer images

\subsection{Isosurface construction}

The $3 \mathrm{D}$ isosurface representation, $\phi(x, y, z), 1 \leq z \leq 2$, can now be expressed as:

$$
\phi(x, y, z)=(z-1) \mathcal{D}_{\mathcal{C}_{2}}(x, y)+(2-z) \mathcal{D}_{\mathcal{C}_{1}}(x, y)
$$

This isosurface $\phi(x, y, z)=0$ is:

$$
z(x, y)=\frac{\mathcal{D}_{\mathcal{C}_{2}}(x, y)-2 \mathcal{D}_{\mathcal{C}_{1}}(x, y)}{\mathcal{D}_{\mathcal{C}_{2}}(x, y)-\mathcal{D}_{\mathcal{C}_{1}}(x, y)}
$$

which is exactly the surface that we reconstructed in (6). From equation (8), since $1 \leq z \leq 2$ if and only if $\mathcal{D}_{\mathcal{C}_{2}}(x, y) \mathcal{D}_{\mathcal{C}_{1}}(x, y) \leq 0, \phi(x, y, z)=0$ occurs only in the region $R\left(\mathcal{C}_{1}, \mathcal{C}_{2}\right)$. Thus, we cannot get artificial isosurface. Figure 3 to Figure 6 show the reconstruction results for different types of $\mathcal{C}_{1}, \mathcal{C}_{2}$ combinations. 


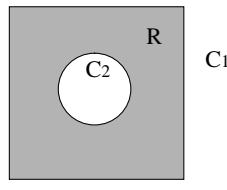

(a)

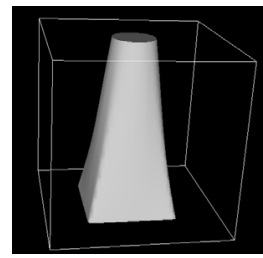

(b)
Figure 3: Surface reconstructed from two contours. contours; (b) computed surface.

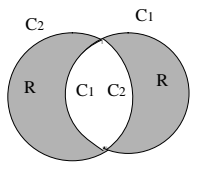

(a)

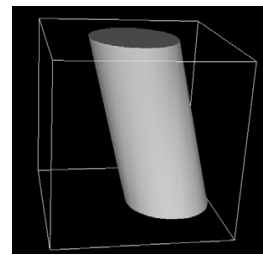

(b)
Figure 4: Surface reconstructed from two contours. contours; (b) computed surface.

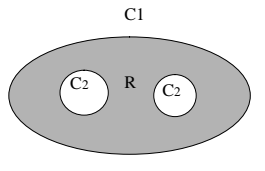

(a)

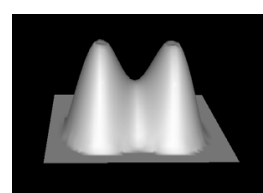

(b)
Figure 5: Surface reconstructed from two contours. contours; (b) computed surface.
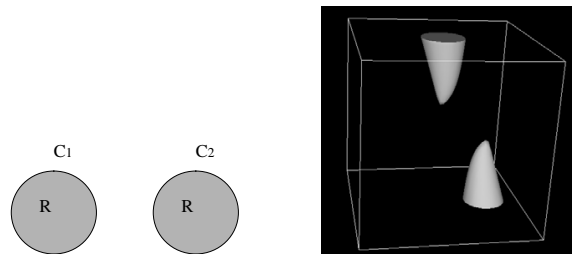

(a)

(b)

Figure 6: Surface reconstructed from two contours. contours; (b) computed surface.

\subsection{Extension to multiple contours}

The proposed method can be applied iteratively to every pair of adjacent contours $\mathcal{C}_{i}, \mathcal{C}_{i+1}, i=1, \ldots, m-1$ for constructing a series of subsurfaces $\mathcal{S}_{i}$. These subsurfaces $\mathcal{S}_{i}, i=1, \ldots, m-1$ form the whole surface, namely $\mathcal{S}=$ $\bigcup_{i} \mathcal{S}_{i}$. Suppose that we want a resolution of $\sigma<1$, say 0.1 , along the $z$ direction, then reconstruction should include between each pair of adjacent contours $Z-1$ new slices with $Z=\frac{1}{\sigma}$ ( a integer $Z$ is expected). The following algorithm produces the $3 \mathrm{D}$ data $\phi(x, y, z), z=1, \ldots, m Z+1$, of which the isosurface $\phi=0$ is $\mathcal{S}$.

Algorithm 1 Construction of the isosurface.

1. Calculate $\mathcal{D}_{\mathcal{C}_{i}}, i=1, \ldots, m$.

2. for $i=1$ to $m-1$, do

$$
\begin{aligned}
& \text { 2.1. for } j=0 \text { to } Z-1, \text { do } \\
& \text { 2.1.1. } z=(i-1) Z+j+1 \text {. }
\end{aligned}
$$

$j \sigma) \mathcal{D}_{\mathcal{C}_{i}}(x, y)$

$$
\text { 2.1.2. } \phi(x, y, z)=j \sigma \mathcal{D}_{\mathcal{C}_{i+1}}(x, y)+(1-
$$

3. End of $i$ loop.

4. $\phi(x, y, m Z+1)=\mathcal{D}_{\mathcal{C}_{m}}(x, y)$

End of the algorithm.

Even for traditional shape recovery

from cross-sectional-contours methods, some post processing(smoothing) is essential to make the reconstructed surface smoother. In our method, if the $\mathcal{C}_{i}$ s are considerably apart from each other in the $x, y$ plane, then the surface may be not smooth on the contour locations. The simplest way to smooth the surface is to convolve $\phi(x, y, z)$ with a 3D Gaussian filter with small scale. Since Gaussian is infinitely differentiable, the smoothed data, and thus the isosurface, is infinitely continuous.

The experimental results are presented in Figure 7 and Figure 8 . We do not perform post-smoothing in these experiments. Figure 7 shows the surface reconstructed from real CT data. The original CT data is $64 \times 64$ at 64 slices, in which the contours correspond to the surface of the bone. We sample the original data along the $z$ direction, at different rates, to form a volume representation that includes 32 , 21 , and 16 slices respectively. These contours are then used for surface reconstruction and interpolation in order to construct the original data set for comparison. Figure 7 (c), (d), and (e) are the reconstructed bone surfaces. Figure 8 shows another reconstruction example from segmented magnetic resonance images of a patient with edema. Segmentation was performed using a Bayesian model and iterative conditioning mode algorithm. For an earlier version of this work, see [21]. This figure shows the white matter corresponding to the cerebral cortex, as well as damaged area due to edema. This original data included 68 slices, and two new slices have been reconstructed from each pair of adjacent slices.

\subsection{A regularized solution for sparse contours}

The result indicates that the proposed technique-a simple linear interpolation-behaves well for dense contours. However, linear interpolation as well as triangulation techniques produce discontinuities for sparse contour representation. This kind of data is often generated in fringes obtained from optical or microwave interferometry $[9,16,15,17]$, and previous methods generate discontinuities along the surface of the object, as shown in Figure 9 (a) and (b). Thus, a more elaborate method to treat this problem is needed. Here, we only consider the situation in which $\mathcal{C}_{i+1}$ is totally enclosed by $\mathcal{C}_{i}$ for $i=1, \ldots, m-1$.

We thus seek a solution for the following problem:

$$
\text { Minimize } \varepsilon^{2}=\iint_{R\left(\mathcal{C}_{1}, \mathcal{C}_{m}\right)} \mathcal{J}_{1}^{2} d x d y
$$




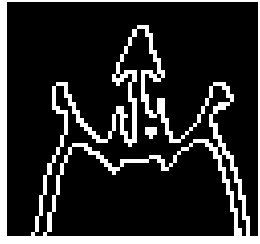

(a)

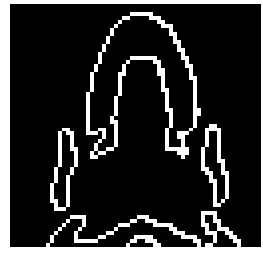

(b)

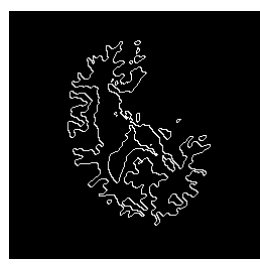

(a)

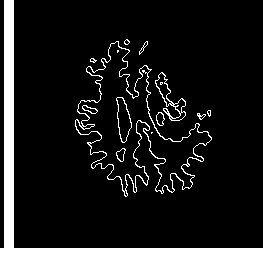

(b)

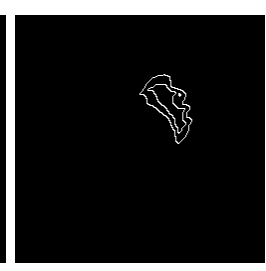

(c)

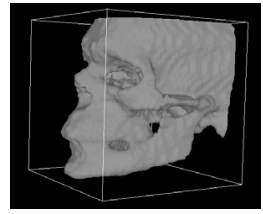

(c)

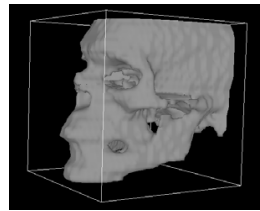

(d)

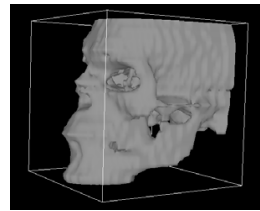

(e)
Figure 7: Reconstruction results from CT data with different sampling rate: (a)(b)Two of the contours; (c)Sample rate 2 ; (d) Sample rate 3 ; and (e)Sample rate 4.

$$
\text { Subject to: } f\left(\mathcal{C}_{i}\right)=i, \quad i=1, \ldots m .
$$

We can use an iterative approach to find the $f$ that minimizes (9):

$$
f^{v+1}=f^{v}-\sigma \delta f^{v}
$$

where $\sigma$ is the step size, $v$ indicates the iteration number and $\delta f$ is the variation of $f$ :

$$
\delta f=-\frac{\partial \mathcal{F}_{f_{x}}}{\partial x}-\frac{\partial \mathcal{F}_{f_{y}}}{\partial y}+\frac{\partial^{2} \mathcal{F}_{f_{x x}}}{\partial x^{2}}+\frac{\partial^{2} \mathcal{F}_{f_{x y}}}{\partial x \partial y}+\frac{\partial^{2} \mathcal{F}_{f_{y y}}}{\partial y^{2}}
$$

where derivatives of $\mathcal{F}$ are defined as:

$$
\begin{aligned}
\mathcal{F}_{f_{x}} & =\frac{\partial \mathcal{J}_{1}^{2}}{\partial f_{x}} \\
& =4 \mathcal{J}_{1} \frac{f_{x} f_{y}^{2} f_{x x}+f_{y}^{3} f_{x y}-f_{x}^{2} f_{y} f_{x y}-f_{x} f_{y}^{2} f_{y y}}{\left(f_{x}^{2}+f_{y}^{2}\right)^{2}} \\
\mathcal{F}_{f_{y}} & =\frac{\partial \mathcal{J}_{1}^{2}}{\partial f_{y}} \\
& =4 \mathcal{J}_{1} \frac{f_{x}^{2} f_{y} f_{y y}+f_{x}^{3} f_{x y}-f_{x}^{2} f_{y} f_{x x}-f_{x} f_{y}^{2} f_{x y}}{\left(f_{x}^{2}+f_{y}^{2}\right)^{2}} \\
\mathcal{F}_{f_{x x}} & =\frac{\partial \mathcal{J}_{1}^{2}}{\partial f_{x x}}=2 \mathcal{J}_{1} \frac{f_{x}^{2}}{f_{x}^{2}+f_{y}^{2}} \\
\mathcal{F}_{f_{x y}} & =\frac{\partial \mathcal{J}_{1}^{2}}{\partial f_{x y}}=4 \mathcal{J}_{1} \frac{f_{x} f_{y}}{f_{x}^{2}+f_{y}^{2}} \\
\mathcal{F}_{f_{y y}} & =\frac{\partial \mathcal{J}_{1}^{2}}{\partial f_{y y}}=2 \mathcal{J}_{1} \frac{f_{y}^{2}}{f_{x}^{2}+f_{y}^{2}}
\end{aligned}
$$

$\delta f$ is set to zero when $\nabla f=0$, indicating that the surface remains unchanged. See Figure $9(\mathrm{c})$ and other experimental results in Figures 10 and 11.

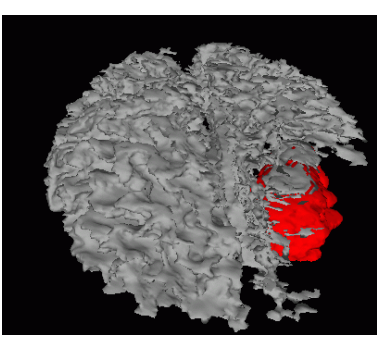

(d)

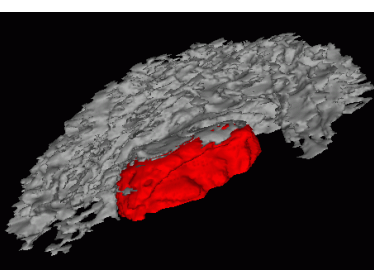

(e)

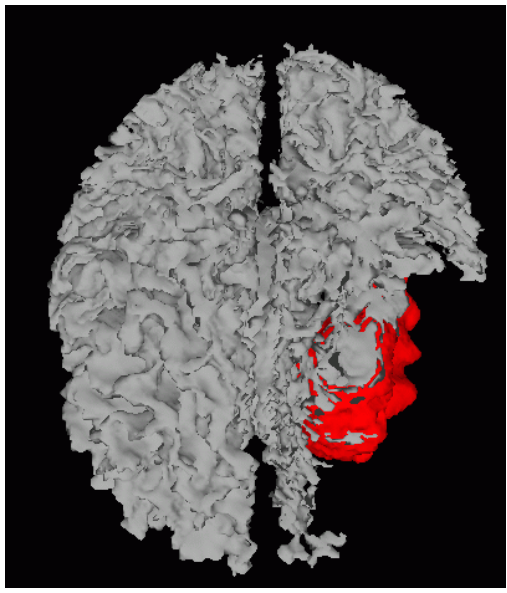

(f)

Figure 8: Reconstruction results of white matter in cortex and region due to edema: (a)(b)(c) Contours from segmented cortex; $(d)(e)(f)$ three views of reconstructed surfaces.

\subsection{Extension to other types of cross sections}

Thus far, we applied our approach to cross sections obtained along the $z$ axis. Other types of $3 \mathrm{D}$ cross sections, as shown in Figure 12(a), can be defined as the intersections of the surface $\mathcal{S}$ with serial of planes along its spine. In this case, we can forward project the $3 \mathrm{D}$ contours to a new coordinate, shown in Figure $12 \mathrm{~b}$, reconstruct the surface, and then back-project the surface to its initial coordinate system.

Figure 13 shows another variation due to a surface of 


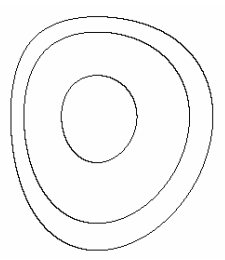

(a)

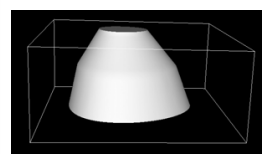

(b)

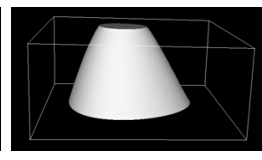

(c)

Figure 9: Reconstruction for sparse contours. (a) three cross-sectional-contours; (b) surface reconstructed by the algorithm 1; (c) surface reconstructed by the regularized solution.

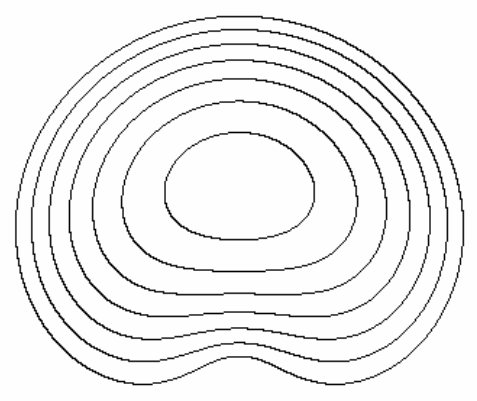

(a)

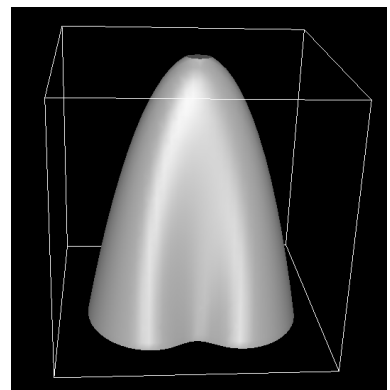

(b)

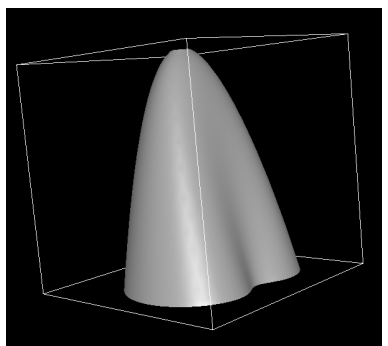

(c)
Figure 10: Reconstruction result: (a) original contours; (b) (c) two views of reconstruction.

revolution. Suppose we rotate a 3D object along a fixed axis and record the cross section perpendicular to each viewing direction. In this case, the contours are the intersections of the $\mathcal{S}$ with a serial of planes rotated along the axis. We can first reconstruct an object by treating the cross sections as if they were in the $z$ direction and then arrange the reconstructed object properly according to the rotating angles.

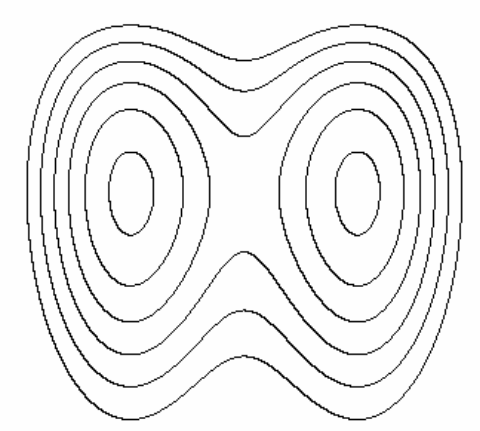

(a)

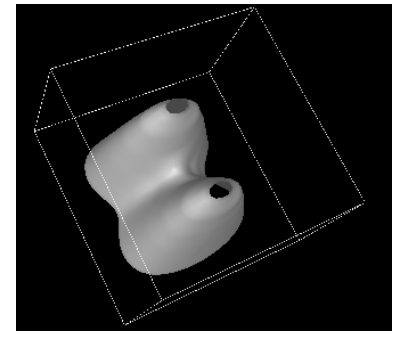

(b)

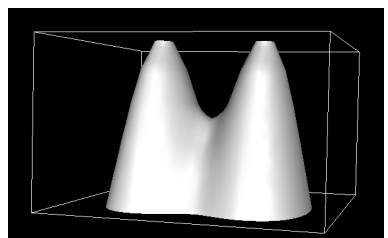

(c)
Figure 11: Reconstruction results: (a) original contours; (b)(c) two views of reconstruction.

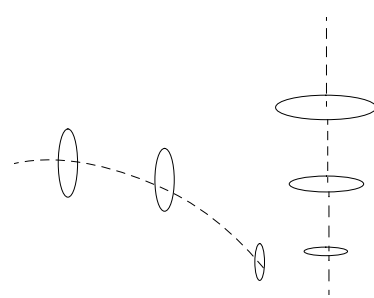

(a)

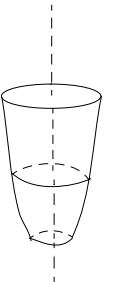

(c)

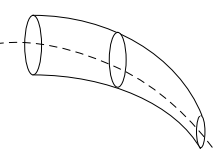

(d)
Figure 12: Reconstruction of cross section along a curve: (a) number of cross sections along a curve; (b) projection of cross sections were in the $z$ direction; (c) reconstruction along the $z$ direction; (d) back projection the reconstructed surface.

\section{Conclusion}

Shape from cross sections is an important problem in diverse fields of science, and it has been studied extensively. However, most (if not all) of these methods suffer from correspondence, tiling, and branching problems. Furthermore, previous solutions have been limited to variants of triangulation that are scale dependent, with unpredictable behavior near the saddle points. The novelty of the pro- 


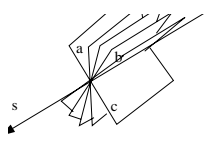

(a)

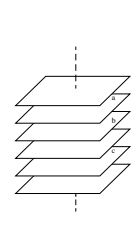

(b)

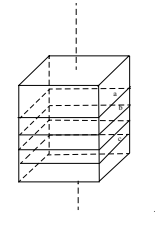

(c)

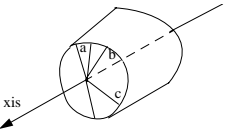

(d)
Figure 13: Reconstruction of surface of revolution: (a) serial sections rotated along an axis; (b) serial sections projected along the $z$ axis; (c) surface reconstruction along the $z$ axis; (d) surface transformation onto original coordinate.

posed method is its unique smoothness measure, the corresponding $\mathrm{PDE}$, and its simple solution based on distance transform. We showed that a linear solution provides an adequate representation of dense contours. In the case of sparse contour, we augmented our approach with a regularized solution to minimize the changes of the gradient. We have tested and verified our approach on data with different degrees of complexities, ranging from simple geometric features to complex and convoluted structure of cortex.

\section{A A note on infinity Laplacian}

This equation is the limit for $p \rightarrow \infty$ of the so-called $p$ Laplace equation, whose solution $f$ minimize the $L_{p}$ norm of the gradient among functions $\phi$ satisfying the given boundary values of $R\left(\mathcal{C}_{1}, \mathcal{C}_{2}\right)$ :

$$
L_{p}(\phi)=\left(\int_{R\left(\mathcal{C}_{1}, \mathcal{C}_{2}\right)}|\nabla \phi|^{2 N} d x d y\right)^{\frac{1}{2 N}}, \quad N \rightarrow \infty
$$

In the limit the $L_{p}$ norm turns into the supermum norm. Formally the solutions of this equation are the "minimizers of the sup norm of the gradient" [1].

\section{References}

[1] G. Aronsson. Extension of function satisfying lipschitz conditions. Arikv för Matematik, 6:551-561, 1967.

[2] G. Aronsson. On the partial differential equation $u_{x}^{2} u_{x x}+2 u_{x} u_{y} u_{x y}+u_{y}^{2} u_{y y}=0$. Arikv för Matematik, 7:133-151, 1968.

[3] G. Aronsson. On certain singular solutions of the partial differential equation $u_{x}^{2} u_{x x}+2 u_{x} u_{y} u_{x y}+u_{y}^{2} u_{y y}=$ 0. Manuscripta Math, 41:133-151, 1981.

[4] C. Bajaj, E. Coyle, and K. Lin. Arbitrary topology shape reconstruction from planar cross sections. Graphical Models and Image Processing, 58(6):524$543,1996$.

[5] J. Boissonnat. Shape reconstruction from planar cross sections. Computer Vision, Graphics, and Image Processing, 44(1):1-29, 1988.

[6] Y. Bresler, J. Fessler, and Macovski. A bayesian approach to reconstruction from imcomplete projections of a multiple object $3 \mathrm{~d}$ domain. IEEE Transactions on Pattern Analysis and Machine Intelligence, 11(8):840-858, 1988.
[7] R. Durikovic, T. Yauchi, K. Kaneda, and H. Yamashita. Shape-based calculation and visualization of general cross-section through biological data. In Proceedings of International Conference on Information Visualization, 1997.

[8] A. Ekoule, F. Peyrin, and C. Odet. A triangulation algorithm from arbitrary shaped multiple planar contours. ACM transactions on graphics, 10(2):182-199, 1991.

[9] C. Elachi. Radar images of the earth from space. Scientific American, 247(6):54-61, December 1982.

[10] L. Evans. Estimates for smooth absolutely minimizing lipschitz extensions. Electronic Journal of Differential Equations, http://ejde.math.swt.edu/Volumes/1993/03Evans/abstr.html, 1993(3):1-9, 1993.

[11] H Fuchs, Z. Kedem, and S. Uselton. Optimal surface reconstruction from planar contours. Communications of the ACM, 20(10):693-702, 1977.

[12] G. Gitlin, J. O'Rourke, and V. De Pigueiredo. On reconstruting polyhedra from parallel slices. International Journal of Computational Geometry and Application, 6(1):103-122, 1996.

[13] E. Keppel. Approximating complex surfaces by triangulation of contour lines. IBM Journal of Research and Development, 19:2-11, 1975.

[14] W. Lin and S. Chen. A new surface interpolation technuque for reconstructing $3 \mathrm{~d}$ objects from serial cross-sections. Computer Vision, Graphics, and Image Processing, 48:124-143, 1989.

[15] J. Marroquin, M. Servin, and R. Rodriguez-Vera. Adaptive quadrature filters and the recovery of phase from fringe pattern images. Journal of Optical Society of American, A, 14(8):1742-1753, August 1997.

[16] D. Massonnet. The displacement field of the landers earthquake mapped by radar interferometry. Nature, 364:138-142, July 1993.

[17] D. Massonnet. Satellite radar interferometry. Scientific American, 276(2):46-53, February 1997.

[18] D. Meyers, S. Skinner, and K. Sloan. Surface from contours. ACM transactions on graphics, 11(3):228$258,1992$.

[19] O. Monga, N. Ayache, and P. Sander. From voxel to intrinsic surface features. Image and Vision Computing, 10(6), 1992.

[20] O. Monga, S. Benayoun, and O. Faugeras. From partial derivatives of $3 \mathrm{~d}$ density images to ridge lines. In Proceedings of the Conference on Computer Vision and Pattern Recognition, pages 354-359, 1992.

[21] B. Parvin, W. Johnston, and D. Robertson. Visualizing volumetric structures from $\mathrm{mr}$ images of the brain. In AAAI Symp. on Application of Computer Vision to Medical Imaging, 1994.

[22] J. Sethian. Curvature and the evolution of fronts. Communications of Mathematical Physics, 101:487499, 1985.

[23] Y. SHinagawa and T. Kunii. The homotopy model: A generalized model for smooth surface generation from cross sectional data. The Visual Computer, $7(2-3): 72-$ 86, 1991 .

[24] Y. Wang and J. Aggarwal. Surface reconstruction and representation of 3-d scenes. Pattern Recognition, $19(3): 197-207,1986$. 